\title{
Inhibition Effects of Nitrones on the Corrosion of Mild Steel in Organic Acid Media
}

\author{
M. Thirumalaikumar ${ }^{*}$ and S. Jegannathan \\ Department of Applied Chemistry, Sri Venkateswara College of Engineering, Pennalur, \\ Sriperumbudur - 602105, India
}

Received 12 November 2009; accepted 2 February 2011

\begin{abstract}
Aromatic nitrones have been synthesized and investigated for their function as inhibitor for the corrosion of mild steel in the organic acid media $-20 \%$ formic acid and $20 \%$ acetic acid at various inhibitor concentrations in the range of $50-150 \mathrm{ppm}$. Weight loss measurements and potentio-dynamic polarization studies reveal that the nitrones behave as mixed type inhibitors. Among the nitrones tested, para-methyl substituted derivatives perform better as inhibitors than the un-substituted nitrones.
\end{abstract}

Keywords: corrosion inhibition, mild steel, adsorption, nitrones.

\section{Introduction}

Studies on the corrosion of metals / alloys in organic acid media are of industrial importance since most carboxylic acids are used as building blocks in a variety of industrial products such as plastics, fibres, drugs, pharmaceuticals, etc. [1-9]. Organic substances with hetero-atoms such as nitrogen, oxygen, sulphur etc., which possess lone pair of electrons, are used as corrosion inhibitors for various combinations of materials and corroding media [10-13]. It has been observed that the adsorption of the inhibitor mainly depends on the presence of electrons on the heteroatom. Adsorption is greatly induced by the inhibitor molecules with higher electron density [14]. The behaviour of nitrones as inhibitors of corrosion of mild steel in acidic solutions has been reported in the literature [15,16]. T.Vasudevan et al. [16] have shown in their study that nitrones function by adsorption following Temkin's model and also by the reduction of hydrogen permeation current. Now, we wish to report an efficient method for corrosion inhibition of mild steel in organic acid media using nitrones.

\footnotetext{
* Corresponding author. E-mail address: mtkumar@svce.ac.in
} 


\section{Experimental}

\section{Synthesis of nitrones}

The nitrones are prepared by the reported method [17-19] and the details of the nitrones used for the study is listed below. The inhibition of corrosion of mild steel in organic acids (such as $20 \%$ formic acid and $20 \%$ acetic acid) are carried out and the results discussed.

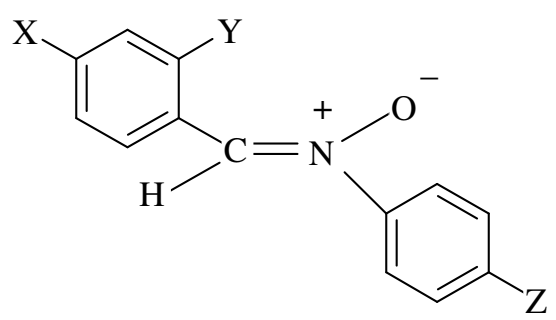

\begin{tabular}{lcccc}
\hline \multicolumn{1}{c}{ Name } & Abbreviation & $\mathbf{X}$ & $\mathbf{Y}$ & $\mathbf{Z}$ \\
\hline$\alpha$, N-diphenyl nitrone & $\mathrm{BN}$ & $\mathrm{H}$ & $\mathrm{H}$ & $\mathrm{H}$ \\
$\alpha$-(4-chlorophenyl)-N-phenyl nitrone & $\mathrm{BCl}$ & $\mathrm{Cl}$ & $\mathrm{H}$ & $\mathrm{H}$ \\
$\alpha$-(2-hydroxyphenyl)-N-phenyl nitrone & $\mathrm{SN}$ & $\mathrm{H}$ & $\mathrm{OH}$ & $\mathrm{H}$ \\
$\alpha$-phenyl-N-(4-methylphenyl) nitrone & $\mathrm{MBN}$ & $\mathrm{H}$ & $\mathrm{H}$ & $\mathrm{CH}_{3}$ \\
$\alpha$-(4-chlorophenyl)-N-(4-methylphenyl) nitrone & $\mathrm{MBCl}$ & $\mathrm{Cl}$ & $\mathrm{H}$ & $\mathrm{CH}_{3}$ \\
$\alpha$-(2-hydroxyphenyl)-N-(4-methylphenyl) nitrone & $\mathrm{MSN}$ & $\mathrm{H}$ & $\mathrm{OH}$ & $\mathrm{CH}_{3}$ \\
\hline
\end{tabular}

\section{Electrolyte}

AR Grade formic acid, acetic acid and double distilled water were used for the preparation of corroding medium / test solution. The inhibitors were synthesized following the procedure described earlier [17-19] and the compounds were characterized through the spectral data and their purity confirmed by TLC / FTIR / NMR study [20,21].

\section{Material preparation}

Weight loss measurement

Mild steel specimens of size $6 \mathrm{~cm} \mathrm{X} 5 \mathrm{~cm} \mathrm{X} 0.05 \mathrm{~cm}$ were used for weight loss measurement. Each coupon was degreased by washing with ethanol followed by rinsing in acetone, allowed to dry and kept in a desiccator prior to use. The precleaned and weighed coupons were dipped in beakers containing $20 \%$ formic and acetic acid by choosing the inhibition concentration of 50 and $150 \mathrm{ppm}$ of nitrones and substituted nitrones, respectively. The immersion time of 24 hours at room temperature was maintained during the experiment. All weight loss measurements are reported as the average of three trials to achieve the accuracy. At the end of the test, the specimen was carefully washed with distilled water, dried and weighed accurately. The weight loss was calculated from the difference between the weight before and after the end of the experiment, and the corrosion rate was calculated using the following formula: 


\section{Corrosion rate $=534 \mathrm{~W} / \mathrm{DAT}$}

where $\mathrm{W}, \mathrm{D}, \mathrm{A}$ and $\mathrm{T}$ are respectively weight loss in $\mathrm{mg}$, density in $\mathrm{g} / \mathrm{cc}$, specimen area in square inch and exposure time in hours [22].

The inhibition efficiency (IE) was calculated using the relation

$$
\mathrm{IE}=\left(\mathrm{W}_{0}-\mathrm{W}_{\mathrm{i}}\right) \mathrm{X} 100 / \mathrm{W}_{0}
$$

where $\mathrm{W}_{0}$ and $\mathrm{W}_{\mathrm{i}}$ are respectively the weight loss values in the absence and in the presence of inhibitor. The weight loss measurements were taken at different concentrations of the various inhibitors.

\section{Adsorption isotherm}

Since the inhibitive action is promoted by adsorption phenomena, the surface coverage $(\theta)$ of the inhibitors is determined using the relation

$$
\theta=\left(\mathrm{W}_{0}-\mathrm{W}_{\mathrm{i}}\right) / \mathrm{W}_{0}
$$

The experiments were carried out as per the method described [23]. The adsorption data are fitted into Temkin adsorption isotherm.

\section{Electrochemical polarization measurements}

Strips of mild steel specimens of the same composition as used for weight loss measurement were used for potentiodynamic polarization studies. The strips were coated with commercially available lacquer with an exposed area of 1.0 $\mathrm{cm}^{2}$. The experiments were carried out at the bath temperature of $20{ }^{\circ} \mathrm{C}$. Autolab Electrochemical Analyzer with three-electrode assembly accessory was used for this study. Platinum foil of $2 \mathrm{~cm} \mathrm{X} 2 \mathrm{~cm}$ size was used as the counter-electrode and a Saturated Calomel Electrode (SCE) was used as reference electrode. The working electrode attained the steady potential - rest potential in the corroding medium within 5 minutes. Both anodic and cathodic polarization studies were made at a sweep rate of $1 \mathrm{mV} / \mathrm{s}$.

\section{Results and discussion \\ Weight loss measurement}

From weight loss measurement, it is evident that the inhibition efficiency increases with increase in concentration of the given inhibitor, in both the acids. The inhibition efficiency tends to decrease more in formic acid than in acetic acid. It has been observed that IE\% of all the substituted nitrones increased when compared to nitrones, and also with increase in concentration from 50 to 150 $\mathrm{ppm}$. The IE\% increased indicating the formation of a film over the surface of mild steel which protects the metal from dissolution. The inhibition efficiency of the inhibitor material depends on various factors, such as the number of adsorption sites, charge density, molecular size, heat of hydrogenation, mode of interaction with the metal surface to form a metallic complex [24]. The inhibitor molecule inhibits corrosion of mild steel by adsorption on the mild steel-solution 
interface; adsorption provides the information about the interaction around the adsorbed molecules themselves as well as their interaction with the metal surface [25]. The inhibition efficiency of nitrones and substituted nitrones in formic and acetic acid with two different concentrations are shown in Fig. 1 and Fig. 2, respectively.

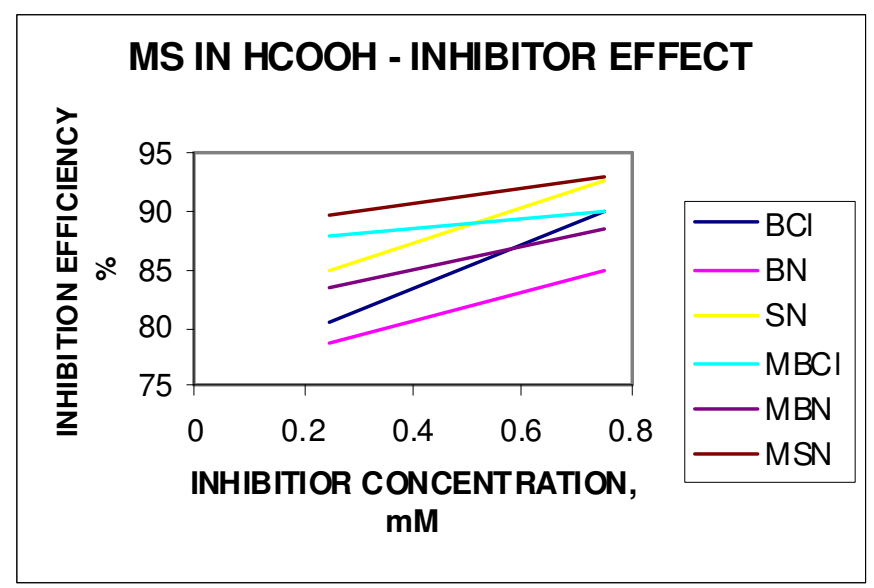

Figure 1. Inhibition efficiency of nitrones and substituted nitrones in $20 \%$ formic acid.

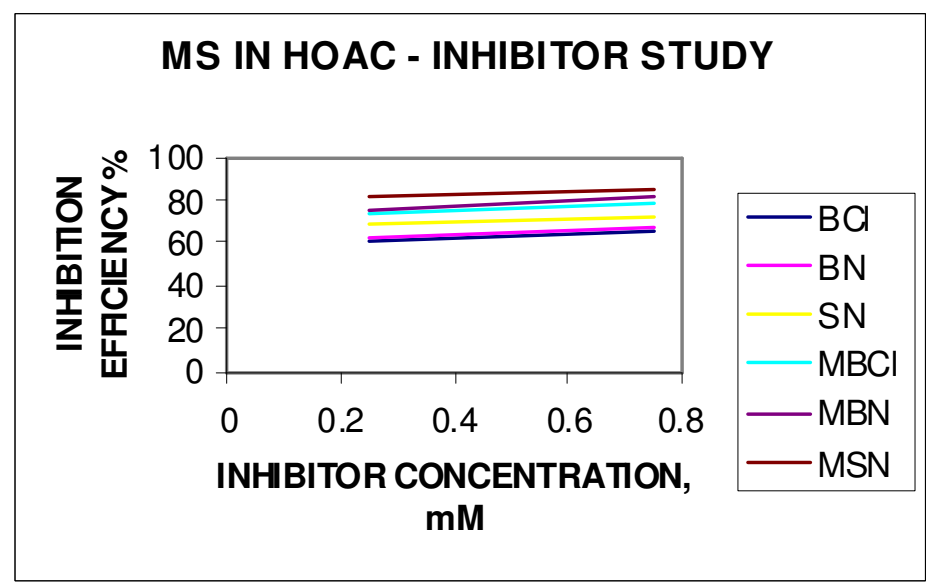

Figure 2. Inhibition efficiency of nitrones and substituted nitrones in $20 \%$ acetic acid.

\section{Adsorption isotherm}

The extent of inhibition depends on the nature and mode of adsorption of the inhibitors on the metal surface [26]. The adsorption follows a quasi-substitution process between water molecules on the surface and organic molecules. The adsorption follows Temkin's model. The degree of surface coverage $\theta$ for nitrones and substituted nitrones in formic acid and acetic acid was evaluated using weight loss method. The data obtained were tested graphically by $\theta$ vs. In C. Fig. 3 and 4 conform the suitability of Temkin isotherm. 


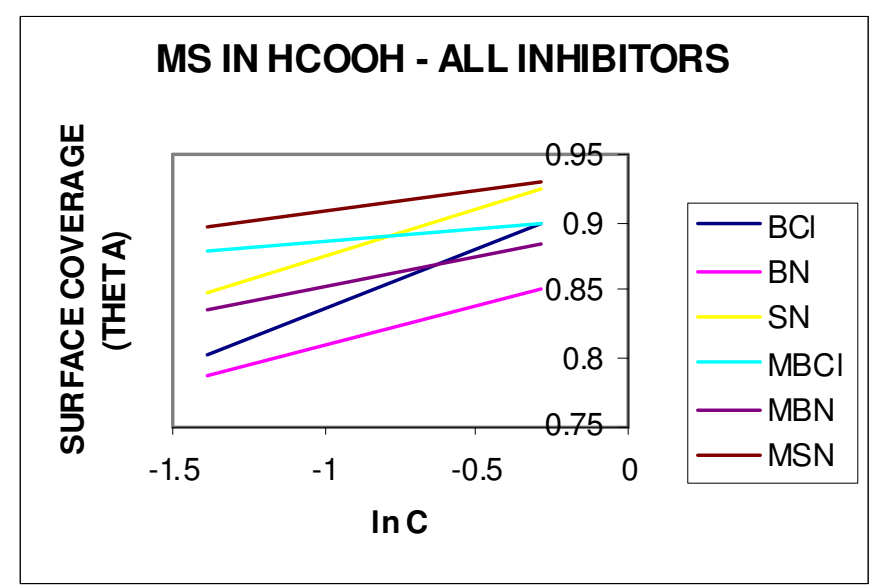

Figure 3. Temkin type isotherm for nitrones and substituted nitrones in $20 \%$ formic acid.

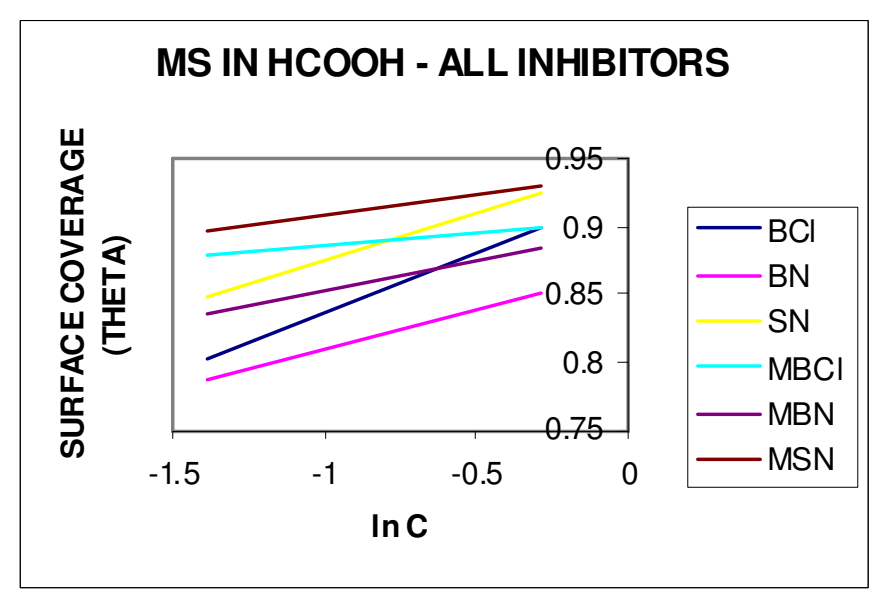

Figure 4. Temkin type isotherm for nitrones and substituted nitrones in $20 \%$ acetic acid.

The sequence of inhibition capacity is of the order $\mathrm{SN}>\mathrm{BCl}>\mathrm{BN}$ in $20 \%$ formic acid medium for unsubstituted nitrones, whereas it follows the order MSN $>\mathrm{MBCl}>\mathrm{MBN}$ for para-methyl substituted derivatives. The overall performance of the para-methyl substituted nitrones in corrosion inhibition is better than that of un-substituted nitrones. This is attributed to the higher electron density on the methyl-substituted nitrones, which enhances the adsorption characteristics with the metal surface and hence promotes inhibition. This observation is further evidenced by the higher surface coverage values $(\theta)$ of substituted nitrones than those of the unsubstituted nitrones.

\section{Electrochemical polarization studies}

Potentiodynamic behaviour of the anodic dissolution of mild steel in acid medium in presence and absence of nitrones is given in Fig. 5 for formic acid and in Fig. 6 for acetic acid. From the polarization curves, it is evident that the inhibitors block the active sites for cathodic reaction to occur and also prevent the metal dissolution process. Furthermore, it is observed that cathodic curves are more affected than anodic curves, indicating that the nitrones act as mixed inhibitors showing predominant cathodic effect. Moreover, the nitrones possess electro-active nitrogen, oxygen atoms and an aromatic ring which favour the 
adsorption phenomenon. The nitrone molecules tend to be adsorbed on to the mild steel substrate as a negatively charged species which can form a complex with the positive metal cation $\mathrm{Fe}^{2+}$. Furthermore, corrosion of mild steel in organic acid medium may occur as follows [9]:

$$
\begin{gathered}
\mathrm{Fe}+\mathrm{HCOO}^{-} \rightarrow[\mathrm{Fe}(\mathrm{HCOO})]_{\mathrm{ads}}+\mathrm{e}^{-} \\
{[\mathrm{Fe}(\mathrm{HCOO})]_{\mathrm{ads}} \rightarrow[\mathrm{Fe}(\mathrm{HCOO})]^{+}+\mathrm{e}^{-}} \\
{[\mathrm{Fe}(\mathrm{HCOO})]^{+}+\mathrm{H}^{+} \rightarrow \mathrm{Fe}^{2+}+\mathrm{HCOOH}}
\end{gathered}
$$

Hydrogen evolution takes place in any acid medium as per the following sequence [16,27]:

$$
\mathrm{M}+\mathrm{H}_{3} \mathrm{O}^{+}+\mathrm{e} \rightarrow \mathrm{H}_{2} \mathrm{O}+\mathrm{MH}_{\mathrm{ads}} ; \mathrm{MH}_{\mathrm{ads}}+\mathrm{MH}_{\mathrm{ads}} \rightarrow 2 \mathrm{M}+\mathrm{H}_{2} \uparrow
$$

Similarly for formic acid medium, which is almost 10 times stronger than acetic acid, the hydrogen evolution can take place on mild steel surface as follows:

$$
\begin{gathered}
\mathrm{Fe}+\mathrm{HCOOH}+\mathrm{e}^{-} \rightarrow \mathrm{FeH}_{\mathrm{ads}}+\mathrm{HCOO}^{-} \\
\mathrm{FeH}_{\mathrm{ads}}+\mathrm{FeH}_{\mathrm{ads}} \rightarrow \mathrm{H}_{2}+\mathrm{Fe}
\end{gathered}
$$

In practice, the gas evolution was observed more in $20 \% \mathrm{HCOOH}$ than in $20 \%$ $\mathrm{AcOH}$. The inhibiting trend of various nitrones in $20 \%$ acetic acid medium is as follows: $\mathrm{SN}>\mathrm{BN}>\mathrm{BCl}$ (un-substituted nitrones) and $\mathrm{MSN}>\mathrm{MBN}>\mathrm{MBCl}$ (paramethyl substituted nitrones)

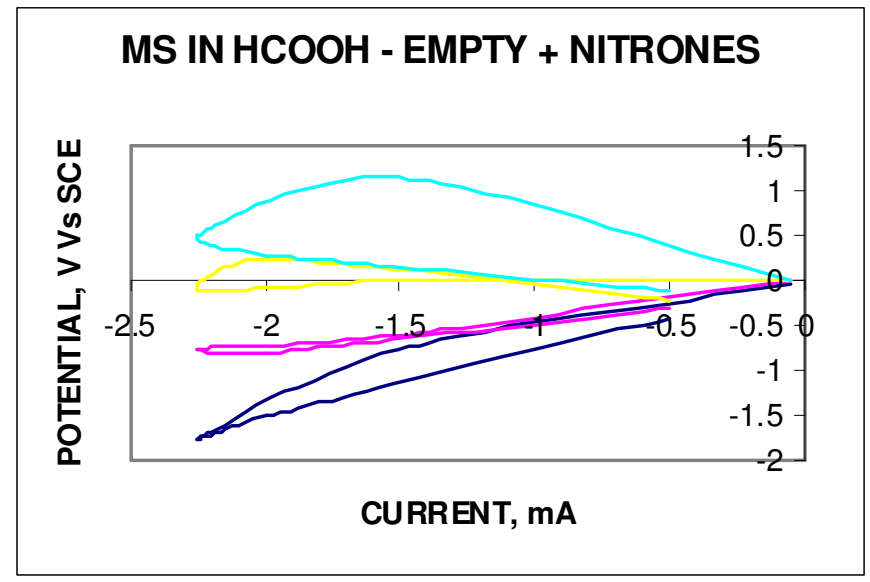

Figure 5. Polarisation curves of mild steel in $20 \%$ formic acid with and without nitrones. 


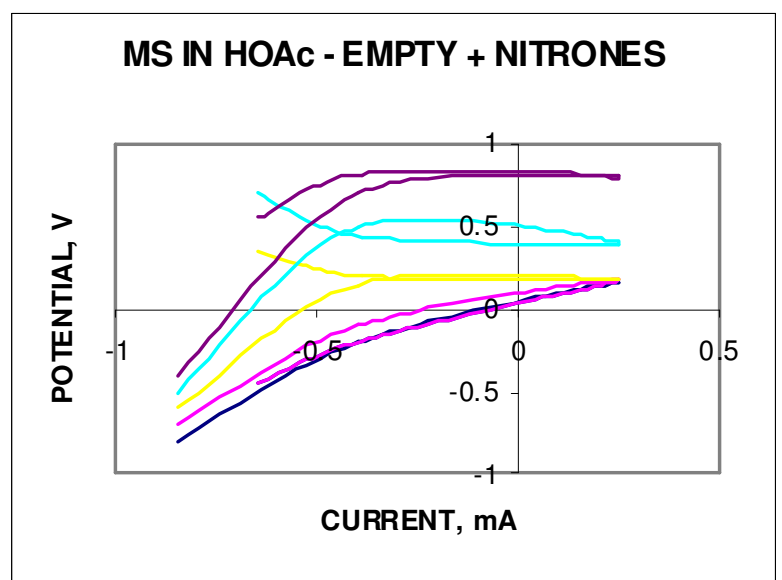

Figure 6. Polarisation curves of mild steel in $20 \%$ acetic acid with and without nitrones.

The overall performance of the para-methyl substituted nitrones in corrosion inhibition is better than that of un-substituted nitrones. This is attributed to the higher electron density on the methyl-substituted nitrones, which enhances the adsorption characteristics with the metal surface and hence promotes inhibition. Here the poor performance of $\mathrm{BCl}$ and $\mathrm{MBCl}$ compared to that in $20 \%$ may be due to the presence of more electronegative chlorine atom, which effectively reduces the electron density of the inhibitor but promotes ionization. The resultant ionic species are in more contact with the ionized acetic acid than being adsorbed on the metal surface to render inhibition characteristics. This observation is further evidenced by the higher surface coverage values $(\theta)$ of substituted nitrones than the unsubstituted nitrones.

\section{Conclusion}

In conclusion, we have shown that nitrones show better performance as inhibitors for corrosion of mild steel in $20 \%$ formic acid and $20 \%$ acetic acid. Methyl substituted nitrones perform better than un-substituted nitrones in inhibiting corrosion. It was found that all the compounds function by adsorption mechanism following Temkin's model.

\section{Acknowledgement}

The authors acknowledge the Principal, Sri Venkateswara College of Engineering, Pennalur, Sriperumbudur for constant encouragement and support provided to carry out this work and the Chief Scientific Officer and his associates, Technology Innovation Centre (SPIC) for the co-operation offered in carrying out the polarization studies.

\section{References}

1. I.A. Sekine, A. Masuko, K. Senoo, Corrosion Sci. 43 (1987) 553.

2. M.A. Quraishi, D. Jamal, Corrosion 56 (2000) 156. 10.5006/1.3280531

3. V.B. Singh, R.N. Singh, Corrosion Sci. 37 (1995) 1399. 10.1016/0010938X(95)00042-I

4. I. Sekine, S. Hatakeyama, Y. Nakazawa, Corrosion. Sci. 27 (1987) 275. 10.1016/0010-938X(87)90023-0 
5. E. Heitz, Corrosion of Metals in Organic Solvents, Plenum Press, New York, NY, (1974) 226.

6. I. Sekine, H. Ohkawa, T. Hank, Corrosion Sci. 22 (1982) 1113. 10.1016/0010-938X(82)90098-1

7. I. Sekine, A. Chinda, Corrosion 40 (1984) 95.

8. M.M. Singh and A. Gupta, Mater. Chem. Phys. 46 (1996) 15. 10.1016/02540584(96)80124-6

9. M.Z.A. Rafiquee, S. Khan, N. Saxena, M.A. Quraishi, Port. Electrochim. Acta 25 (2007) 419. 10.4152/pea.200704419

10. S. Muralidharan, M.A. Quraishi, S.V.K. Iyer, Anti-Corr. Methods Mater. 44 (1997) 100. 10.1108/00035599710165342

11. M.A. Quraishi, M.A.W. Khan, M. Ajmal, Anti-Corr. Methods Mater. 43 (1996) 5.

12. B. Hammouti, A. Aouniti, M. Taleb, M. Bright, S. Kertit, Corrosion 51 (1995) 411. 10.5006/1.3293606

13. A.-E. Nabey, E. Khammis, M. Sh. Ramadan, A.E. Gindy, Corrosion 52 (1996) 671. 10.5006/1.3292157

14. M.M. Osman, R.A. El-Ghazawy, A.M. Al-Sabagli, Mater. Chem. Phys. 80 (2003) 55. 10.1016/S0254-0584(01)00588-0

15. R. Hariharaputran, A. Subramanian, A.A. Antony, P. Manishankar, A. Gopalan, T. Vasudevan, S.V.K. Iyer, British Corrosion J. 33 (1998) 214.

16. R. Hariharaputran, A. Subramanian, A.A. Antony, P. Manishankar, T. Vasudevan, S.V.K. Iyer, S. Anti- Corr. Methods Mater. 46 (1999) 35. 10.1108/00035599910252785

17. S. Sivasubramanian, C. Amutha, M. Thirumalaikumar, S. Muthusubramanian, Indian J. Chem. 35(B) (1996) 503.

18. M. Thirumalaikumar, S. Sivasubramanian, A. Ponnuswamy, P. Mohan, Eur. J. Med. Chem. 31 (1996) 905. 10.1016/S0223-5234(97)89854-6

19. P. Merino, P. Padar, I. Delso, M. Thirumalaikumar, T. Tejero, L. Kovacs, Tetrahedron Lett. 47 (2006) 5013. 10.1016/j.tetlet.2006.05.114

20. J. Hamer, A. Macaluso, Chem. Rev. 64 (1964) 473. 10.1021/cr60230a006

21. N. Arumugam, P. Manisankar, S. Sivasubramanian, D.A. Wilson, Magn. Reson. Chem. 23 (1985) 246. 10.1002/mrc.1260230407

22. S. Acharya, S.N. Upadhyay, Trans. Indian Inst. Met. 57 (2004) 297.

23. M. Sahin, B. Bilgic, H. Yilmaz, Appl. Surf. Sci. 195 (2002) 1. 10.1016/S0169-4332(01)00783-8

24. N. Hackerman, E.S. Snavely, J.S. Payne, J. Electrochem. Soc. 113 (1966) 677. 10.1149/1.2424089

25. A.K. Dubey, G. Singh, Port. Electrochim. Acta 25 (2007) 205. 10.4152/pea.200702205

26. S. Muralidharan, R. Chandrasekar, S.V.K Iyer, Proc. Indian. Acad. Sci. (Chem. Sci.) 112 (2000) 127. 